\title{
Evaluation of Vestibular Evoked Myogenic Potentials (VEMP) Individuals with Tinnitus and Normal Hearing
}

Ayhan Admis ${ }^{1}$

Selim Unsal ${ }^{2 \star}$ Mehmet Gunduz ${ }^{3}$

\begin{abstract}
The aim the present study is to evaluate ocular and cervical Vestibular Evoked Myogenic Potentials (VEMPs) of adult individuals with and without tinnitus who have normal hearing. Thirty one patients who have normal hearing, complaining about unilateral or bilateral subjective tinnitus and 30 volunteers who without tinnitus and vestibular complains were enrolled into the present study. Following examination by of Ear Nose Throat (ENT) physician, Tinnitus Disability Questionnaire (TDQ), pure tone audiometry $(125-16.000 \mathrm{~Hz})$ speech tests, oVEMP and cVEMP tests were applied to all participants. Fourteen individuals with normal hearing and tinnitus were male $(44 \%)$ and $17(56 \%)$ were female with an age average of $35.8 \pm 10.2 .30$ years; the control group consisted of 16 males $(53 \%)$ and 14 females (47\%) with an age average of $37.5 \pm 12$ years. Two individuals of the tinnitus group had bilateral tinnitus and 29 individuals had unilateral tinnitus. A statistically significant difference in individuals without tinnitus and tinnitus must be normal at work between test answers that are not available $125-16000 \mathrm{~Hz}$ range Air Conduction (AC) hearing thresholds, speech tests, cVEMP and oVEMP responses. Tinnitus may appear due to many reasons. Vestibular system is one of the conditions that can cause tinnitus. According to the findings obtained from adult individuals with normal hearing, the vestibular system does not cause tinnitus.
\end{abstract}

Keywords: Normal hearing; tinnitus; cVEMP; oVEMP; participants. 


\section{INTRODUCTION}

Tinnitus, a ringing in the corresponding word is derived from the Latin word "tinnire", which means to ring the bell ${ }^{1}$. Tinnitus is not a disease only, but also a reflection of a pathology in the hearing system ${ }^{1,2}$. However, tinnitus may become a more important problem than underlying pathology. Tinnitus is basically classified as subjective or objective ${ }^{1-3}$. Patients with objective tinnitus are heard from the external environment and assistive listening devices. In subjective tinnitus, sound it is only perceived by the patient ${ }^{1-4}$. There are many factors which contribute to the formation and have role in the hearing system. However, it is usually difficult to determine how and why tinnitus occurs ${ }^{1}$. While the most common known cause of hearing loss with tinnitus formation there are many situations that are associated with tinnitus. Although there are many individuals with hearing loss and without tinnitus, individuals who have normal hearing also may develop complaint of tinnitus ${ }^{5,6}$. Noise exposure, obesity, smoking and alcohol use, head trauma, arthritis, hypertension and various pharmacological agents may be considered among the risk factors. Tinnitus disease may occur alone or along with other symptoms of Meniere's Disease (MD), vestibular migraine, acoustic neuroma and otosclerosis. In this disease, abnormal VEMP responses may be monitored ${ }^{5-7}$. Recently begun to be widely used VEMP test otolith organs of the muscle stimulation results in ending reflex response that is measured in electrophysiological test methods ${ }^{8}$. The reflex response measured from extraocular muscles is called ocular Vestibular Evoked Myogenic Potentials (OVEMP), and from sternocleidomastoid (SKM) muscle is called cervical VEMP (cVEMP). Cervical VEMP responses when assessing the saccule and inferior vestibular nerve function, oVEMP response largely on utriculi and is believed to evaluate the superior vestibular nerve function ${ }^{5}$. The aim of the present study, subject tinnitus in normal hearing individuals with tinnitus that may affect the vestibular otolith formation is to evaluate the presence of system induced pathology.

\section{MATERIALS AND METHODS}

The consent for the study was obtained from Human Research Ethics Committee with decision number of 99950669\136 on 28.05.2015 and the present study was conducted in Audiology and Speech Pathology Clinic of a tertiary medical Centre. All patients whom Ear Nose Throat (ENT) examination was performed and subjective tinnitus was detected were included into the study. Thirty-one adult individuals with tinnitus who have normal hearing consisted of 14 males (44\%) and 17 females (56\%) with an age average of $35.8 \pm 10.2$. Thirty individuals included in the control group consisted of males $16(53 \%)$ males and $14(47 \%)$ females with an age average of $37.5 \pm 12$. Two of them were in the bilateral tinnitus group and 29 adults had unilateral tinnitus. Thirteen (41.9\%) individuals had tinnitus on the right ear whereas 16 (51.6\%) individuals had tinnitus on the left ear and $2(6.5 \%)$ had tinnitus on both ears. Duration of complaint of tinnitus varied between 6 and 120 months with average duration of 19 months. All participants were exposed to tinnitus disability questionnaire, pure tone audiometry, speech tests, oVEMP and cVEMP tests individuals have middle ear pathology, neurological problems, head trauma and vestibular complaints which were excluded from the study. Pure tone audiometry test double hull was made quite cabin with Interacoustics AC-40 clinic audiometry (Interacoustics Assens, Middelfart, Denmark). TDH-39 headphones for Air Conduction (AC) measurements and KOSS HV-IA headphones for high frequency measurement (8.000-16.000 Hz) are used. Bone measurements were performed with a Radio Ear B71 V vibrator. Giving voice to the microphone for Speech Reception Threshold (SRT), Word Discrimination Score (WDS) and Uncomfortable Loudness (UCL) were determined. Electroacoustic impedance tests were performed through Interacoustics AZ26 (Interacoustics Assens, Middelfart, Denmark). A TDH-39 speaker and +200 daPa with -400 daPa pressure and a probe at a tone of $226 \mathrm{~Hz}$ were used. In the range of \pm 100 decaPascal (daPa) middle ear pressure values were accepted as normal value (Tip A). It was recorded using oVEMP and cVEMP tests Otometrics ICS Charter EP200 System (Otometrics GN, Taastrup, Denmark). In the range of $10 \mathrm{~Hz}-1500 \mathrm{~Hz}$ EMG signals were amplified and filtered. Stimuli were provided with ER3A insert headphones (Etymotic Research Inc, Illinois, USA). The frequency of $500 \mathrm{~Hz}$ tone burst stimulus intensity 4\s in $100 \mathrm{dBnHL}$ was given. Registration was limited to 200 alerts. Negative (rarefaction) polarity was used. During testing of the electrode impedance, it was noted to be less than 5000 ohms. Skin cleansing gel was applied before EEG a disposable surface electrode was employed. The active electrode was located on $1 \backslash 3$ of the SCM muscle, the reference electrode was located on a part of the sternum, and the ground electrode was located onto the forehead for cVEMP measurements. SCM contraction was provided in supine position with the head of one right turn in front of the stimulated side. SCM contractions that occur in the ipsilateral muscle myogenic inhibitor activity was measured on the right and left ear. The active electrode was located onto the infraorbital rime, the reference electrode was located just $2-2.5 \mathrm{~cm}$ below the ground electrode was located onto the forehead for oVEMP measurements. The participants were asked to look at on predetermined object at supine position; the eyes were provided in the horizontal axis forming an angle of 30-40 degrees. The stimuli were given to the contralateral eye and recorded with insert earphones. That reaction occurring when the stimulus given in cVEMP, the first positive wave $\mathrm{P} 1$ and subsequent negative wave $\mathrm{N} 1$ were determined. In oVEMP, N1 and P1 were determined following administration of the stimuli consisting of wave form peaks.

Statistical Analysis: A statistical Package for Social Science (SPSS) for Windows 20.0 software was used for statistical analysis. To find relationships between 
variables the difference between the means test has been application. The statistically significance level was accepted as the difference between means below 0.05 .

\section{RESULTS}

Air conduction hearing thresholds in both groups at $0.125-16 \mathrm{kHz}$ was obtained in the normal range, and statistically significant differences were not detected $(p>0.05)$. Six adults in the tinnitus group and 5 adults in the control group over 40 years of age presented an increase when compared to normal hearing threshold at 8-16 $\mathrm{kHz}$ frequency range. Furthermore, in the tinnitus group, Air Conduction (AC) hearing thresholds were compared between 33 ears with tinnitus and 29 years without tinnitus; and statically significant differences were observed $(p>0.05)$ (Table 1). Tinnutus group and the control group were compared for speech tests. Tinnutus group of 31 years and the control group of 60 ears obtained Speech Reception Threshold and Uncomfortable Loudness were shown in Table 2. SRT and UCL values were within normal limits in the tinnitus and control groups. A Statistically significant difference was observed $(p>0.05)$. In the tinnitus group, significant differences were observed between the ears with and without tinnitus $(p>0.05)$. Tinnitus group evaluated 62 ears (33 with tinnitus, 29 without tinnitus) in 6 indivudals UCL values in the ears was tinnitus showed a lower level of intensity. cVEMP responses were compared separately for both left and right ears in the tinnitus and control groups; and statiscally significant differences were observed $(p>0.05)$. There were statically significant differences in cVEMP responses between 33 ears with tinnitus and 29 years without tinnitus $(p>0.05)$ Asymetry rate was obtained in the normal range for both groups. There was not any statistically significant difference between the groups $(p>0.05)$ (Table 3$)$. oVEMP responses were compared seperately for both left and right ears the tinnitus group and the control group and statistically significant differences were observed $(p>0.05)$. Tinnitus in groups of 2 indivudals (unilateral), the control groups of 1 individual's oVEMP could not to be obtained. There were statistically significant differences between 31 ears with tinnitus and 58 ears in the control group $(p>0.05)$. Ocular VEMP responses were compared between 31 ears with tinnitus and 27 years without tinnitus and statistically significant differences were not detected $(p>0.05)$ (Table 4). Tinnitus Disability Questionnaire (TDQ) grading total score in 14 individuals 2 nd degree ( $46 \%$ middle), in 10 individuals $3 \mathrm{rd}$ (32\% moderate), in 2 individuals 4 th degree $(6 \%$ severe), in 5 individuals 5 th degree $(16 \%$ disaster) scores were determined in severity. The average total score TDQ was Grade 3.

\section{DISCUSSION}

In recent years, several studies were conducted on conditions with tinnitus in otoneurology. Etiology and pathophysiology of tinnitus was not yet fully understood and studies still continue in this regard ${ }^{8}$. Tinnitus was a symptom of many visual outward from a disease pathology. The pathology occur anywhere in the auditory system, it can lead to an increase of the sound perceived with unknown mechanism ${ }^{9,10}$. It was reported the

Table 1. Air Conduction (AC) hearing thresholds in groups.

\begin{tabular}{|c|c|c|c|c|}
\hline \multicolumn{5}{|c|}{ Group } \\
\hline \multirow[t]{2}{*}{ Frequency } & $\begin{array}{l}\text { Tinnitus Group } \\
(n=31)\end{array}$ & $\begin{array}{l}\text { Control Group } \\
(n=30)\end{array}$ & $\mathbf{t}$ & p value \\
\hline & Mean \pm SD & Mean \pm SD & & \\
\hline Right 0.125 kHz & $9.3 \pm 3.3$ & $9 \pm 4.6$ & 0.342 & $0.734^{*}$ \\
\hline Right $0.250 \mathrm{kHz}$ & $8.3 \pm 4.5$ & $7.8 \pm 5.3$ & 0.436 & $0.665^{\star}$ \\
\hline Right 0.500 kHz & $9 \pm 4.3$ & $8.8 \pm 5.2$ & 0.162 & $0.872^{\star}$ \\
\hline Right $1 \mathrm{kHz}$ & $8 \pm 4.7$ & $7.1 \pm 5.3$ & 0.691 & $0.492^{*}$ \\
\hline Right 2 kHz & $8.3 \pm 5.5$ & $7.5 \pm 5.8$ & 0.609 & $0.545^{*}$ \\
\hline Right 4 kHz & $8.2 \pm 4.5$ & $7.5 \pm 6.5$ & 0.501 & $0.618^{*}$ \\
\hline Right 6 kHz & $8.3 \pm 6.8$ & $7.8 \pm 5.6$ & 0.342 & $0.733^{*}$ \\
\hline Right 8 kHz & $11.2 \pm 7.8$ & $12 \pm 7.3$ & -0.364 & $0.718^{*}$ \\
\hline Right 12 kHz & $19.0 \pm 17.8$ & $18.1 \pm 13.9$ & 0.211 & $0.834^{*}$ \\
\hline Right 16 kHz & $23 \pm 17$ & $24.1 \pm 15.4$ & -0.265 & $0.792^{*}$ \\
\hline Left $0.125 \mathrm{kHz}$ & $9.1 \pm 4.4$ & $8 \pm 4.6$ & 1.019 & $0.313^{*}$ \\
\hline Left $0.250 \mathrm{kHz}$ & $8.2 \pm 3.9$ & $7 \pm 6.1$ & 0.925 & $0.359 *$ \\
\hline Left $0.500 \mathrm{kHz}$ & $8.5 \pm 4.3$ & $7.3 \pm 5$ & 1.012 & $0.316^{\star}$ \\
\hline Left $1 \mathrm{kHz}$ & $8.39 \pm 4.9$ & $7.1 \pm 6.2$ & 0.850 & $0.399 *$ \\
\hline Left $2 \mathrm{kHz}$ & $5.6 \pm 4.9$ & $6.6 \pm 5.7$ & -0.742 & 0.461 * \\
\hline Left $4 \mathrm{kHz}$ & $8.9 \pm 5.2$ & $8.5 \pm 6.1$ & 0.276 & $0.784^{*}$ \\
\hline Left $6 \mathrm{kHz}$ & $9.0 \pm 6.2$ & $8.1 \pm 9.2$ & 0.430 & $0.671^{*}$ \\
\hline Left 8 kHz & $12.1 \pm 9.2$ & $12.1 \pm 10.2$ & -0.028 & $0.978 *$ \\
\hline Left $12 \mathrm{kHz}$ & $18.7 \pm 17.6$ & $17.5 \pm 15$ & 0.287 & $0.775^{\star}$ \\
\hline Left $16 \mathrm{kHz}$ & $21.4 \pm 16.5$ & $22.6 \pm 14$ & -0.309 & $0.759 *$ \\
\hline
\end{tabular}


Table 2. Speech test results.

\section{Speech Test Results (dB HL)}

Right SRT

Left SRT

Right UCL

Left UCL

\section{Group}

$\begin{gathered}\text { Tinnitus Group } \\ (\mathbf{n}=\mathbf{3 1})\end{gathered}$
Mean \pm SD
$13 . \pm 2.7$
$13.2 \pm 3$
$107.1 \pm 4.2$
$106.9 \pm 4.7$

$\begin{gathered}\text { Control Group } \\ (\mathbf{n}=\mathbf{3 0})\end{gathered}$
Mean \pm SD
$12.8 \pm 3.1$
$12.1 \pm 3.3$
$107.3 \pm 3.8$
$107.5 \pm 3.8$

$\mathrm{p}$ value

$0.762^{*}$

$0.204^{*}$

$0.821^{*}$

$0.615^{\star}$

Table 3. Comparison of cVEMP test results.

\section{Comparison of cVEMP Test Answers}

Right P1 latency (msec)

Right N1 latency (msec)

Right P1-N1 Amplitude $(\mu \mathrm{V})$

Right P1-N1 Interval (msec)

Left P1 latency (msec)

Left N1 latency (msec)

Left P1-N1 Amplitude $(\mu \mathrm{V})$

Left P1-N1 Interval (msec)

Asymmetry Rate (\%)

*The difference between two average Materiality Test; SD: Standard Deviation

\begin{tabular}{|c|c|c|c|}
\hline \multicolumn{4}{|l|}{ Group } \\
\hline & $\begin{array}{l}\text { Control Group } \\
\qquad(n=30)\end{array}$ & $\mathbf{t}$ & $p$ value \\
\hline \multicolumn{4}{|c|}{ Mean \pm SD } \\
\hline & $15 \pm 1.2$ & 1.460 & $0.150^{*}$ \\
\hline & $23.2 \pm 2.1$ & 2.244 & $0.059^{*}$ \\
\hline & $391.5 \pm 157.9$ & 0.018 & $0.986^{*}$ \\
\hline & $8.1 \pm 1.5$ & 2.054 & $0.054^{*}$ \\
\hline & $14.9 \pm 1.1$ & 0.646 & $0.521^{*}$ \\
\hline & $23.7 \pm 2.6$ & 0.601 & $0.550^{*}$ \\
\hline & $391.2 \pm 191.4$ & 0.030 & $0.976^{*}$ \\
\hline & $8.7 \pm 2.2$ & 0.363 & $0.718^{*}$ \\
\hline & $7.8 \pm 6$ & 0.051 & $0.960 *$ \\
\hline
\end{tabular}

Table 4. Comparison of oVEMP test results.

\section{Comparison of oVEMP Test Results}

Right P1 latency (msec)

Right N1 latency (msec)

Right P1-N1 interval (msec)

Left P1 latency (msec)

Left $\mathrm{N} 1$ latency (msec)

Left P1-N1 Interval (msec)

Asymmetry Rate (\%)

*The difference between two average Materiality Test; SD: Standard Deviation

$\begin{array}{lll}\begin{array}{c}\text { Group } \\ \text { Control Group } \\ (\mathbf{n}=29)\end{array} & \mathbf{t} & \\ \quad \begin{array}{c}\text { Mean } \pm \text { SD } \\ 1.1\end{array} & & \\ 10.5 \pm 1.1 & -1.006 & 0.319^{*} \\ 16.1 \pm 1.11 & -0.637 & 0.527^{*} \\ 5.5 \pm 1.2 & 0.985 & 0.329^{*} \\ 10.5 \pm 0.4 & -0.863 & 0.393^{\star} \\ 15.8 \pm 0.9 & -0.795 & 0.430^{*} \\ 5.28 \pm 0.8 & -0.160 & 0.874^{\star} \\ 11.2 \pm 5.4 & -0.658 & 0.513^{*}\end{array}$

the groups in terms of Air Conduction hearing thresholds range $0.125-16 \mathrm{kHz}, \mathrm{SRT}, \mathrm{UCL}$, WDS values in comparison with responses of cVEMP and OVEMP. In audiology evaluation of 11 individuals older than 40 years of age, the increase in bilateral high-frequency hearing threshold was considered as the beginning of presbycusis. Tinnitus 6 individuals UCL values tinnitus ear at a lower intensity level is to be observed in these individual's cochlea suggest that the affected physiological levels. Two individuals in the tinnitus group and 1 individual in the control group with inability to obtain the oVEMP response were associated with the pathogenesis of tinnitus. Vestibular disorders are non-specific symptoms such as vertigo, tinnitus, dizziness, feeling of fullness in the ears and hearing loss. Other symptoms may occur alone with tinnitus such as Meniere's disease, Vestibular migraine, acoustic neuromas and otosclerosis. In this disease, 
abnormal VEMP responses may be monitored ${ }^{5-7}$. Herraiz et al. $^{16}$ stated that $25 \%$ of the patients with Meniere's reported tinnitus as the initial symptom ${ }^{16}$ Havia et al. ${ }^{17}$ detected tinnitus as the initial symptom by $5 \%{ }^{17}$. Robertson et al. ${ }^{18}$ performed cVEMP in the patients diagnosed with Meniere and obtained no response ${ }^{18}$. De Waele et al. ${ }^{19}$ implemented CVEMP in the patients diagnosed with Meniere and no response was obtained by $54 \%{ }^{19}$. VEMP test may be used for diagnostic purposes during earlay stages of Meniere's syndrome ${ }^{20,21}$. Shepard VEMP test with the diagnosis of Meniere's disease may occur in the future could be reported early ${ }^{22}$. Studies considering, in our study in tinnitus patients with tinnitus it has come to mind as Meniere's initial symptoms observed. However, in these patients, suggesting no evidence of Meniere VEMP. In the study of Marcelli et al. ${ }^{23}$, the children with and without vestibular complaints diagnosed with migraine were exposed to audio vestibular tests. Vestibular abnormalities were detected in $73 \%$ and $33 \%$ of the group with and without vestibular complaints, respectively ${ }^{23}$. In the study of Baier and Dieterich ${ }^{23}$ where CVEP was implemented on the patients with vestibular migraine, according to the amplitudes bilateral vestibular migraine in $68 \%$ of patient in the control group it was found to be lower ${ }^{6}$. In the study conducted by Liao and Young ${ }^{24}$, unilateral or bilateral abnormal cVEMP values were found in $50 \%$ of patients with basilar migraine. Based on these findings, the researchers, working on inner ear as well as vestibular brainstem pathology of migraine in particular stated that the affected structures such as saccule and they may contribute to appearance of the symptoms such as vertigo ${ }^{6}$. There was no evidence of ocular or cervical VEMP suggesting migraine was detected in the present study. In the present study, OVEMP responses of both groups were evaluated in 116 years in total and the mean latency of $\mathrm{N} 1$ was $10.45 \pm 0.79 \mathrm{~ms}$ and $\mathrm{P} 1$ latency was $15.85 \pm 0.99 \mathrm{~ms}$. In the cVEMP responses, $\mathrm{P} 1$ latency was $14.99 \pm 1.16 \mathrm{~ms}$, and $\mathrm{N} 1$ latency was $23.88 \pm 2.22 \mathrm{~ms}$. The mean values obtained were found to be consistent with P1 (P13) and N1 (P23) values in the literature ${ }^{20-25}$. Berry et al. ${ }^{26}$ did not find any relationship between Tinnitus Disability Questionnaire (TDQ) scores and pure voice mean and speech discrimination scores. However, $60 \%$ of the cases reported that they were uncomfortable with the loud voice and there was a relationship between the total TDQ score and this parameter ${ }^{27}$. Since our study included individuals with normal hearing, there was no relationship between TDQ scores and Pure Tone Average (PTA) and speech discrimination thresholds. Although it was not statistically significant, 6 individuals with tinnitus had a decrease in Disturbing Sound Level (DSL) in the tinnitus ear compared to normal. When the TDQ scores of these 6 individuals were examined, 4 of them were catastrophic and 2 of them had severe tinnitus.

\section{CONCLUSION}

There is not any difference between pure voice hearing thresholds, SRT, WDS, UCL, OVEMP and cVEMP responses in tinnitus and control groups. In addition, no significant difference was found in the comparison of ears with and without tinnitus within the tinnitus group. It was considered that ocular and cervical VEMP responses could be used in the investigation of tinnitus and the pathological VEMP responses were not observed. It was concluded that the autolytic organs function normally. More studies are needed.

\section{CONFLICT OF INTEREST}

The Author declares no potential conflict of interest on publishing this paper.

\section{REFERENCES}

1. Meyerhoff W, Cooper J. Tinnitus. Otolaryngol. 1991;2:1169-79.

2. Baguley D, Mcferran D, Hall D. Tinnitus. The Lancet. 2013;382:16007.

3. Sharma A, Bakshi J, Munja S. Tinnitus Treatment with Misoprost: A Blinded and Placebo Controlled Study. International Tinnitus Journal. 2018;22:143-9.

4. Nondahl DM, Cruickshanks KJ, Huang GH, Klein BE, Klein R, Nieto FJ, et al. Tinnitus and its risk factors in the Beaver Dam offspring study. Int J Audiol. 2011;50:313-20.

5. Timmer FC, Zhou G, Guinan JJ, Kujawa SG, Herrmann BS, Rauch SD. Vestibular Evoked Myogenic Potential (VEMP) in patients with Ménière's disease with drop attacks. Laryngoscope. 2006;116:7769.

6. Baier B, Dieterich $M$. Vestibular-evoked myogenic potentials in "vestibular migraine" and Menière's disease: A sign of an electrophysiological link Ann N Y Acad Sci. 2009;1164:324-7.

7. Murofushi T, Matsuzaki M, Mizuno M. Vestibular evoked myogenic potentials in patients with acoustic neuromas. Arch Otolaryngol Head Neck Surg. 1998;124:509-12.

8. Akkuzu G, Akkuzu B, Ozluoglu LN. Vestibular evoked myogenic potentials in benign paroxysmal positional vertigo and Meniere's disease. Eur Arch Otorhinolaryngol. 2006;263:510-7.

9. House JW. "Tinnitus: Evaluation and Treatment". Otol Neurotol. 1984;5:472-5.

10. Davis A, Rafaie EA. "Epidemiology of Tinnitus". Tinnitus Handbook. 2000:1-23.

11. Meyerhoff W, Cooper JC, Paparella MM, Shumrick DA, Gluckman Jl, Meyerhoff WL. Tinnitus. In: Eds. Otolaryngology, 3rd Edition. 1991:1169-79.

12. Meikle M, Taylor WE. Characteristics of tinnitus and related observations in over 1800 tinnitus clinic patients. J Laryngol Otol Suppl. 1984;9:17-21.

13. Hazell JW, Wood SM, Cooper HR, Stephens SD, Corcoran AL, Coles RR, et al. A clinical study of tinnitus maskers. $\mathrm{Br} \mathrm{J}$ Audiol. 1985;19:65-146.

14. Dauman R, Cazals Y. Auditory frequency selectivity and tinnitus. Arch Otorhinolaryngol. 1989;246:252-5.

15. Jastreboff P. et al. "Otolaryngology-Head\&Neck Surgery". Otolaryngology Head\&Neck Surgery, 1998.

16. Herraiz C, Tapia MC, Plaza G. Tinnitus and Ménière's disease: characteristics and prognosis in a tinnitus clinic sample. Eur Arch Otorhinolaryngol. 2006;263:504-9.

17. Havia M, Kentala E, Pyykkö I. Hearing loss and tinnitus in Menieres disease. Auris Nasus Larynx. 2002;29:115-9. 
18. Robertson DD, Ireland DJ. Vestibular evoked myogenic potentials. J Otolaryngol. 1995;24:3-8.

19. Waele DC, Tran Ba HP, Diard JP, Freyss G, Vidal PP. Saccular dysfunction in Ménière's patients. A vestibular-evoked myogenic potential study. Ann N Y Acad Sci. 1999;871:392-7.

20. Cal R, Bahmad Jr F. Vestibular evoked myogenic potentials: An overview. Braz J Otorhinolaryngol. 2009;75:456-62.

21. Murofushi T, Shimizu K, Takegoshi H, Cheng PW. Diagnostic value of prolonged latencies in the vestibular evoked myogenic potential. Arch Otolaryngol Head Neck Surg. 2001;127:1069-72.

22. Shepard NT. Differentiation of Ménière's disease and migraineassociated dizziness: A review. J Am Acad Audiol. 2006;17:69-80.

23. Marcelli VI, Furia T, Marciano E. Vestibular pathways involvement in children with migraine: A Neuro Otological Study. Headache. 2010;50:71-6.

24. Liao LJ, Young YH. Vestibular evoked myogenic potentials in basilar artery migraine. Laryngoscope. 2004;114:1305-9.

25. Basta D, Todt I, Ernst A. Normative data for P1/N1-latencies of vestibular evoked myogenic potentials induced by air or boneconducted tone bursts. Clin Neurophysiol. 2005;116:2216-9.

26. Berry JA, Gold SL, Frederick EA, Gray WC, Staecker H. Patientbased outcomes in patients with primary tinnitus undergoing tinnitus retraining therapy. Arch Otolaryngol Head Neck Surg. 2002;128:1153-7.

27. Wu HJ, Shiao AS, Yang YL, Lee GS. Comparison of short tone burstevoked and click-evoked vestibular myogenic potentials in healthy individuals. J Chin Med Assoc. 2007;70:159-63. 